\title{
A role for microRNA in cystic liver and kidney diseases
}

\author{
Andrew S. Chu and Joshua R. Friedman \\ Department of Pediatrics, Division of Gastroenterology, Hepatology and Nutrition, University of Pennsylvania School of Medicine, \\ Children's Hospital of Philadelphia, and The Joseph Stokes, Jr. Research Institute, Philadelphia, Pennsylvania, USA.
}

\begin{abstract}
The polycystic liver and kidney diseases are a family of disorders with heterogeneous etiologies. Proposed mechanisms of disease include ciliary dysfunction, excess cell proliferation, and altered cell-cell or cell-matrix interactions. In this issue of the JCI, Lee and colleagues provide data to support a novel mechanism for cystogenesis involving microRNA (miRNA) (see the related article beginning on page 3714). They demonstrate that levels of the miRNA miR15a are decreased in livers of patients with autosomal recessive and autosomal dominant polycystic kidney disease (ARPKD and ADPKD, respectively) and congenital hepatic fibrosis as well as in the PKC rat model of ARPKD. This results in increased expression of the cell-cycle regulator Cdc25A, which is a direct target of miR15a, and increased cellular proliferation and cystogenesis in vitro. These findings suggest that other miRNAs may also participate in the molecular pathogenesis of cystic liver and kidney diseases.
\end{abstract}

Modern medicine's understanding of disease mechanisms received a surprising gift in 1993 with the first report of a microRNA (miRNA), lin-4, and its role in directing the transition of Caenorbabditis elegans from larval to adult forms (1). Since that time, it has become clear that miRNA plays an important role in the regulation of cell behavior in all animals; this contrasts with older views of RNA as simply the middleman between DNA and protein (2). miRNAs alter protein expression through RNA interference, either by silencing mRNA translation or by increasing mRNA degradation $(3,4)$. It is believed that up to one-third of all protein-coding genes are regulated by miRNAs (5).

The influence of miRNA on fundamental cellular processes such as proliferation and apoptosis has found immediate applicability in cancer research, with miRNA profiling now providing tools for diagnosis as well as for understanding the mechanisms of malignancy (6). However,

Nonstandard abbreviations used: ADPKD, autosomal dominant polycystic kidney disease; ARPKD, autosomal recessive polycystic kidney disease; $\mathrm{Cdc} 25 \mathrm{~A}$, cell division cycle 25A; CHF, congenital hepatic fibrosis; miRNA, microRNA; PKD1, polycystin 1; PKHD1, polycystic kidney and hepatic disease 1 .

Conflict of interest: The authors have declared that no conflict of interest exists.

Citation for this article: J. Clin. Invest. 118:3585-3587 (2008). doi:10.1172/JCI36870. it is clear that miRNA is important in the pathophysiology of a wide range of nonmalignant disorders. In this issue of the $J C I$, Lee and colleagues address the previously unexplored arena of miRNA involvement in the cystogenesis of polycystic liver and kidney diseases (7).

\section{Pathogenesis of polycystic liver and kidney diseases}

The polycystic liver and kidney diseases are a family of disorders with heterogeneous etiologies and a range of phenotypic presentations. Autosomal dominant polycyswith renal and liver cystogenesis that clinically manifests in adulthood, often leading to dialysis and renal transplantation. It is caused by mutations in either of two genes, PKD1 and PKD2, which code for polycystin 1 and polycystin 2 , respectively (8). Autosomal recessive polycystic kidney disease (ARPKD) can present in neonates with massive renal cysts, causing respiratory failure secondary to abdominal competition that subsequently leads to infant demise, although milder forms can present later in life. While ARPKD is always found in conjunction with congenital hepatic fibrosis (CHF), it is less commonly associated with hepatic cystogenesis, and it is linked to mutations in the gene polycystic kidney and hepatic disease 1 (PKHD1), which encodes fibrocystin, another cilitic kidney disease (ADPKD) is associated ary protein $(8,9)$. While the products of PKD1, PKD2, and PKHD1 are present in multiple subcellular locations, one common denominator that they all share (also shared by other genes mutated in other human cystic diseases and animal models) is expression in the cilium. This has led to the theory that cystogenesis is due, at least in part, to ciliary dysfunction (10).

Various theories of cystogenesis have circulated over time, one of the earliest being that excessive proliferation by cells lining renal and biliary ducts leads to redundancy that allows cyst formation. Alterations in apoptosis have also been observed in the cystic liver and kidney diseases (10). Because cilia are, among other things, signal transducers capable of sending messages via a variety of pathways, the aforementioned discovery of mutations in genes encoding ciliary proteins has led to speculation that abnormal ciliary function leads to disordered signal transduction, altered gene expression, and/or alterations in cell-cell and cell-matrix adhesion (10-12).

Specifically, signaling by cilia may occur through any of several nonexclusive pathways. Mechanical stimulation of the motile cilia of renal tubular epithelial cells results in $\mathrm{Ca}^{2+}$ flux across the plasma membrane, followed by the release of intracellular $\mathrm{Ca}^{2+}$ stores, which may result in multiple secondary messenger signals. Together, PKD1 and PKD2 can induce the cell-cycle inhibitor p21/WAF1 and the antiproliferative helix-loop-helix transcription factor ID2 (10). Cells lining cysts have also been found to express elevated levels of EGFR on their apical membranes, supporting the concept of increased cell proliferation as a cause of cyst growth (13). To further complicate the picture, a recent study using an inducible mouse knockout of $P k d 1$ demonstrated a critical time point early in life that determines kidney cyst formation, and the authors of this study did not find a significant elevation of cell proliferation beyond this time 

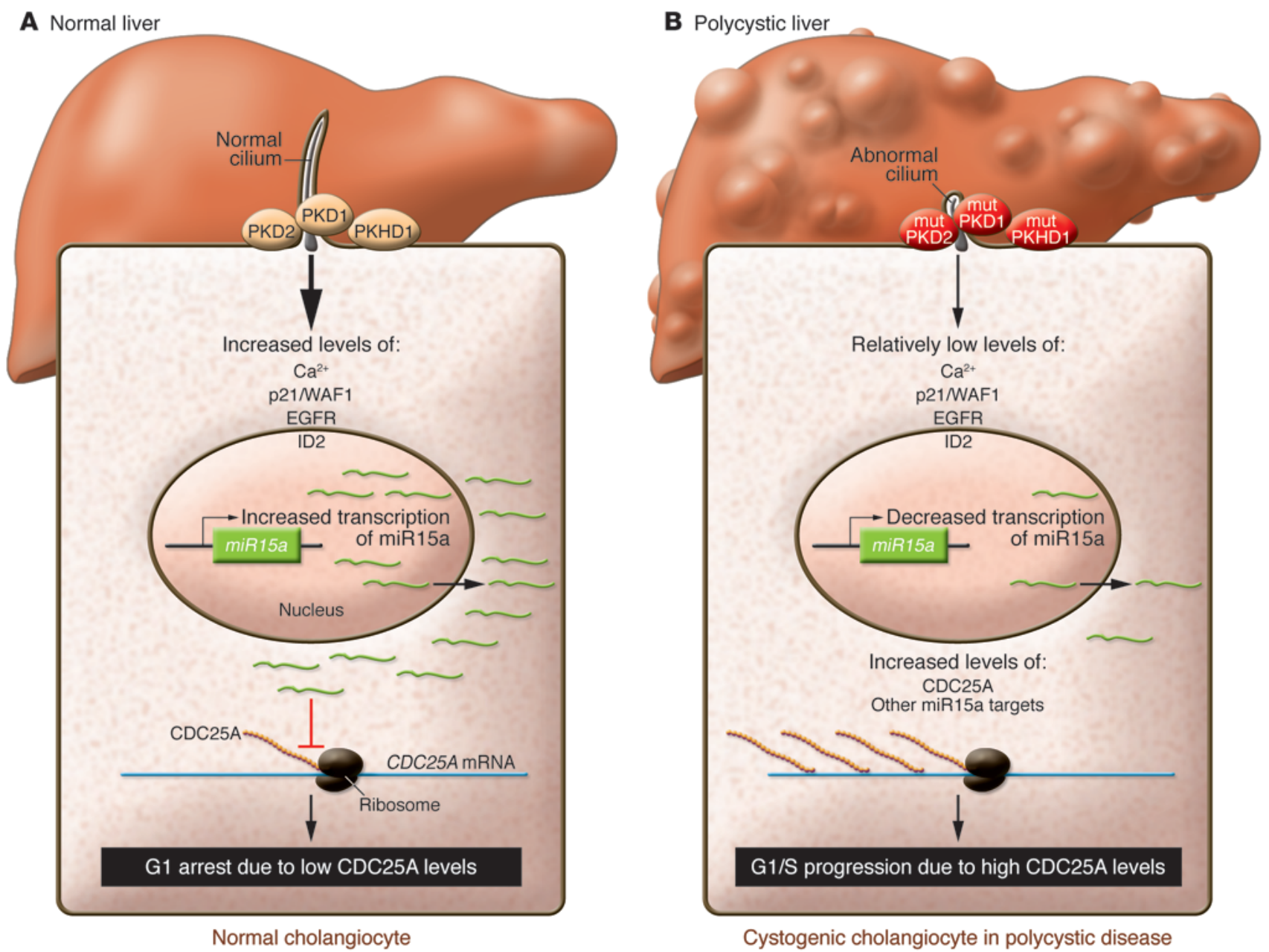

Figure 1

Model of miR15a function in hepatic cystogenesis. (A) In normal cholangiocytes, ciliary signaling results in increased levels of intracellular $\mathrm{Ca}^{2+}, \mathrm{p} 21 / \mathrm{WAF} 1$, EGFR (in the cell membrane), and nuclear ID2 (10). These and/or other events result in miR15a expression and CDC25A repression. Decreased levels of CDC25A then lead to G1 arrest, preventing cyst formation. (B) As demonstrated in this issue of the $J C l$ by Lee and colleagues (7), miR15a levels are reduced in PCK rat cholangiocytes and in ARPKD, CHF, and ADPKD liver specimens. In the PCK rat, ARPKD, and CHF, the $P K D H 1$ gene is mutated [mutPKDH1], while in ADPKD, either $P K D 1$ or $P K D 2$ is mutated. CDC25A levels are increased in these same tissues. Using antisense oligonucleotide knockdown of miR15a in rat cholangiocytes, Lee and colleagues confirmed that reduction of miR15a results in elevated Cdc25A levels, increased cell proliferation, and cyst growth in vitro, while overexpression of miR15a in PCK cholangiocytes has the opposite effect.

point compared with controls, in opposition to several previous studies (14). The proliferation of cells lining hepatic cysts was not measured in that study.

\section{miR15a has an impact on cystogenesis through Cdc25A}

In their study in this issue of the JCI (7), Lee and colleagues significantly contribute to the literature not only by demonstrating increased proliferation of cholangiocytes (bile duct epithelial cells) isolated from the $P C K$ rat (an established rat model of ARPKD) but also by providing a plausible mechanism for cystogenesis involving miRNA (Figure 1) $(15,16)$. After docu- menting that unmodified PCK-CCL cells (a cholangiocyte cell line generated from PCK rats) proliferate at twice the rate of controls, the authors performed miRNA microarray analysis on normal cholangiocyte and PCK-CCL lines (7). Their findings paint a picture of dramatically altered miRNA expression, with PCKCCL cells differentially expressing 109 miRNAs, most of which were downregulated compared with their expression in normal cholangiocytes. The expression of one miRNA in particular, miR15a, was significantly more decreased than that of any other miRNA. Confirmatory experiments on the microarray data demonstrated con- sistent decreases in miR15a expression in both PCK-CCL cells and isolated PCK rat cholangiocytes compared with controls.

While these results are intriguing, their clinical relevance is established by the demonstration of analogous patterns of expression in human disease specimens (7). Comparing normal human liver with liver samples from patients with ADPKD, ARPKD, and CHF, Lee and colleagues used in situ hybridization to show that miR15a levels appeared to be reduced in affected human tissues. Future studies will need to confirm this observation via quantitative analysis, such as quantitative RT-PCR of RNA isolated from human cystic disease specimens. 
Having shown that miR15a levels are lower in cystic liver tissues, the next question is how this observation relates to a specific pathologic mechanism. The answer to this question requires three criteria to be fulfilled: (a) at least one candidate target mRNA is identified; (b) experimental modification of miR15a is reproducibly associated with alterations in the levels of the candidate; and (c) this change is associated with downstream effects upon end points that can plausibly result in cystogenesis. Lee and colleagues used in silico miRNA target prediction to focus on cell division cycle $25 \mathrm{~A}$ (Cdc25A), a member of the Cdc25 family of phosphatases that are critical for progression of the cell cycle (6). Specific targeting of the Cdc25A 3'-UTR by miR15a was confirmed by luciferase reporter assay. Consistent with the repressive effect of miRNA, the authors found that $\mathrm{Cdc} 25 \mathrm{~A}$ protein levels were twice as high in PCK rat cholangiocytes as in controls (7). They then employed confocal immunofluorescence microscopy to show that in human ARPKD, ADPKD, and CHF specimens, cells lining cysts appeared to express higher levels of CDC25A protein than cells lining normal bile ducts. As is the case with miR15a expression in diseased tissues, further studies will be needed to quantify this difference. It will also be important to determine whether the same patterns of miR15a and CDC25A expression are present in the cells lining kidney cysts in ADPKD and ARPKD.

To establish a causal link between miR15a, Cdc25A, and the growth of cysts, the authors utilized antisense oligonucleotides to knock down miR15a in normal rat cholangiocytes (7). They found that anti-miR15a treatment resulted in a small decrease in miR15a and commensurate increases in Cdc25A protein, cellular proliferation, and the growth of cysts in vitro (6). Conversely, overexpression of miR15a in cells derived from the $P C K$ rat resulted in decreased Cdc25A protein, small decreases in G1-S phase transition and cellular proliferation, and a larger drop in cyst growth in vitro. This disproportionate effect on cyst growth suggests that decreased miR15a may promote cystogenesis through mechanisms aside from increased cell proliferation.

\section{Linking miRNA to the polycystic disease phenotype}

As a whole, the findings presented by Lee and colleagues indicate that changes in miRNA expression contribute to the phenotypic changes found in cystic liver disease (7). In the most critical assays - cell proliferation and cyst growth in vitro - the effect of modulating miR15a in normal and PCK rat cholangiocytes was significant but small. This is not surprising in light of the many other miRNAs whose expression is altered in PCK rat cholangiocytes. Nevertheless, several important implications follow. We believe that this is the first direct evidence of the role of miRNA in polycystic liver and kidney diseases. With miRNA already finding use in hepatology, by providing a deeper understanding of hepatic malignancies, hepatitis $\mathrm{C}$, and metabolic regulation, this report adds another area of potential miRNA-based therapy (17).

As with any novel study, the article by Lee and colleagues leaves us with many questions remaining to be answered. Could miRNA play a role in the other potential mechanisms of cystogenesis (mechanosensation, cell-cell and cell-matrix adhesion, planar cell polarity, and others)? What is the etiology of decreased miR15a expression in cystic disease? Could abnormal signaling by dysfunctional cilia drive miRNA dysregulation? What are the roles of the other miRNAs identified by the authors' microarray analysis? The answers to these questions are likely to expose other regulatory pathways involved in cystic liver and kidney diseases.

\section{Acknowledgments}

The authors are grateful to Daniel Weinblatt and Ari Berg for thoughtful reading of the manuscript. A. Chu is a National Institute of Child Health and Human Development (NICHD) fellow of the Pediatric Scientist Development Program and is supported by NICHD grant K12HD000850. J.R. Friedman is supported by NIH grants K08DK070881 and R03DK081450 and the Fred and Suzanne Biesecker Pediatric Liver Center.

Address correspondence to: Joshua R. Friedman, Department of Pediatrics, Division of
Gastroenterology, Hepatology and Nutrition, University of Pennsylvania School of Medicine, Children's Hospital of Philadelphia, The Joseph Stokes, Jr. Research Institute, ARC 1007B, 3615 Civic Center Boulevard, Philadelphia, Pennsylvania 19104-4318, USA. Phone: (267) 426-7223; Fax: (206) 984-2191; E-mail: friedmaj@ mail.med.upenn.edu.

1. Lee, R.C., Feinbaum, R.L., and Ambros, V. 1993. The C. elegans heterochronic gene lin-4 encodes small RNAs with antisense complementarity to lin-14. Cell. 75:843-854.

2. Zhang, C. 2008. MicroRNomics: a newly emerging approach for disease biology. Physiol. Genomics. 33:139-147.

3. Du, T., and Zamore, P.D. 2005. microPrimer: the biogenesis and function of microRNA. Development. 132:4645-4652.

4. Mattick, J.S., and Makunin, I.V. 2006. Non-coding RNA. Hum. Mol. Genet. 15:R17-R29.

5. Xie, X., et al. 2005. Systematic discovery of regulatory motifs in human promoters and 3' UTRs by comparison of several mammals. Nature. 434:338-345.

6. Cho, W.C. 2007. OncomiRs: the discovery and progress of microRNAs in cancers. Mol. Cancer. 6:60.

7. Lee, S.-O., et al. 2008. MicroRNA15a modulates expression of the cell-cycle regulator $\mathrm{Cdc} 25 \mathrm{~A}$ and affects hepatic cytogenesis in a rat model polycystic kidney disease. J. Clin. Invest. 118:3714-3724.

8. Tahvanainen, E., Tahvanainen, P., Kaariainen, H., and Hockerstedt, K. 2005. Polycystic liver and kidney diseases. Ann. Med. 37:546-555.

9. Shneider, B.L., and Magid, M.S. 2005. Liver disease in autosomal recessive polycystic kidney disease. Pediatr. Transplant. 9:634-639.

10. Ibraghimov-Beskrovnaya, O., and Bukanov, N. 2008. Polycystic kidney diseases: from molecular discoveries to targeted therapeutic strategies. Cell. Mol. Life Sci. 65:605-619.

11. Kim, E., and Walz, G. 2007. Sensitive cilia set up the kidney. Nat. Med. 13:1409-1411.

12. Torres, V.E., and Harris, P.C. 2006. Mechanisms of disease: autosomal dominant and recessive polycystic kidney diseases. Nat. Clin. Pract. Nephrol. 2:40-55; quiz 55.

13. Avner, E.D. 1993. Epithelial polarity and differentiation in polycystic kidney disease. J. Cell Sci. Suppl. 17:217-222.

14. Piontek, K., Menezes, L.F., Garcia-Gonzalez, M.A., Huso, D.L., and Germino, G.G. 2007. A critical developmental switch defines the kinetics of kidney cyst formation after loss of Pkd1. Nat. Med. 13:1490-1495.

15. Masyuk, T.V., et al. 2004. Biliary dysgenesis in the PCK rat, an orthologous model of autosomal recessive polycystic kidney disease. Am. J. Pathol. 165:1719-1730.

16. Muff, M.A., et al. 2006. Development and characterization of a cholangiocyte cell line from the PCK rat, an animal model of autosomal recessive polycystic kidney disease. Lab. Invest. 86:940-950.

17. Girard, M., Jacquemin, E., Munnich, A., Lyonnet, S., and Henrion-Caude, A. 2008. miR-122, a paradigm for the role of microRNAs in the liver. J. Hepatol. 48:648-656. 